REVISTA SCIENTIA AGRARIA

Versão On-line ISSN 1983-2443

Versão Impressa ISSN 1519-1125

SA vol. $18 \mathrm{n}^{\circ} .1$ Curitiba Jan/Mar. 2017 p. 83-89

\title{
BALANÇO HÍDRICO CLIMATOLÓGICO E CLASSIFICAÇÃO CLIMÁTICA PARA O MUNICÍPIO DE BALSAS-MA
}

Water balance climatological and climate classification for municipality of Balsas- $M A$

Mádilo Lages Vieira Passos ${ }^{1}$, Geraldo Cesar Zambrzycki², Reginaldo Sérgio Pereira ${ }^{3}$

${ }^{1}$ Graduando em Engenharia Agrícola - CCAA, UFMA, e-mail: madilolages@hotmail.com.

2 Professor, Centro de Ciências Exatas e Tecnologia - CCET, UFMA, e-mail: alvinegro_2@hotmail.com.

${ }^{3}$ Professor Doutor, Departamento de Engenharia Florestal - PGEFL, UnB, e-mail: reginaldosp@unb.br.

Artigo enviado em 21/09/2016, aceito em 28/03/2017 e publicado em 11/04/2017.

\begin{abstract}
RESUMO: O balanço hídrico climatológico (BHC) é uma das formas de monitorar o armazenamento de água no solo, sendo ferramenta essencial no planejamento estratégico agrícola. Objetivou-se com este estudo desenvolver o BHC de acordo com a metodologia proposta por Thornthwaite e Mather (1955), para o município de Balsas-MA e a classificação climática segundo Thornthwaite (1948). Nesse estudo foi utilizada uma série de dados históricos entre os anos de 1976 (início de operações da estação meteorológica no município) a 2015, contendo a precipitação pluvial média mensal e temperatura. Foram desconsiderados dados que, por alguma razão, não foram registrados. Para o cálculo do BHC foi adotado o valor de $100 \mathrm{~mm}$ para a capacidade de água disponível (CAD). A classificação climática foi obtida por meio dos valores do índice hídrico (Ih), índice de aridez (Ia) e índice de umidade (Iu). A evapotranspiração real atingiu 1099 mm e verificou-se um déficit hídrico anual de $621 \mathrm{~mm}$, distribuído em sua totalidade entre os meses de abril e novembro, indicando a necessidade de irrigação nessa época para os cultivos agrícolas. O código climático da região pela metodologia de Thornthwaite foi $\mathrm{C}_{1} \mathrm{dA} \mathrm{A}^{\prime}$ '.
\end{abstract}

Palavras-chave: classificação climática, deficiência hídrica, evapotranspiração.

\begin{abstract}
The climatological water balance (BHC) is one of the ways to monitor the storage of water in the soil, being an essential tool in strategic planning agricultural. The aim of this study to develop the BHC in accordance with the methodology proposed by Thornthwaite and Mather (1955), for the municipality of Balsas (MA) and the Thornthwaite climate classification second (1948). In this study was used a series of historical data from 1976 (beginning of operations at the weather station in the city) to 2015 , containing the pluvial precipitation and average monthly temperature. Were disregarded data that for some reason were not registered. For the calculation of the BHC was adopted the value of $100 \mathrm{~mm}$ for the available water capacity (CAD). The Climate classification obtained by means of the values of the water index (hi), index of aridity (Ia) and moisture index (Iu). The real evapotranspiration reached $1099 \mathrm{~mm}$ and an annual water deficit of $621 \mathrm{~mm}$, distributed in your entirety between the months of april and november, indicating the need for irrigation at this time for agricultural cultivation. The climate of the region code by Thornthwaite methodology was $\mathrm{C}_{1} \mathrm{dA}$ 'a'.
\end{abstract}

Keywords: climate classification, deficiency hydric, evapotranspiration. 


\section{INTRODUÇÃO}

O monitoramento da dinâmica agroclimática é crucial para a otimização da produção agrícola, pois anomalias climáticas são as principais causadoras de baixas na produtividade agrícola mundial.

Segundo Castro (2012), a agricultura nordestina possui grande variabilidade, tanto nas culturas exploradas, quanto na tecnologia empregada para a produção agrícola, o que aliado à irregularidade climática, como os anos de secas e chuvas intensas que se alternam de formas erráticas, dificulta o desenvolvimento, levando à deterioração do solo e da água. Isso evidencia uma melhor compreensão do padrão climático das localidades do Nordeste.

De acordo com Blain (2009) o monitoramento climático é importante para o planejamento da agricultura, pois é a base para o sucesso produtivo das culturas agrícolas, uma vez que determina a melhor época e as áreas mais promissoras ao plantio.

O método de estimativa do balanço hídrico climatológico (BHC) proposto por Thornthwaite e Mather (1955) é uma ferramenta de monitoramento de armazenamento de água no solo, largamente utilizada como instrumento de planejamento estratégico agrícola no âmbito do gerenciamento dos recursos hídricos.

Santos et al. (2010) afirmam que o conhecimento das variáveis estimadas no $\mathrm{BHC}$ favorecem o planejamento agropecuário e as práticas de controle de produção, porque são informações que permitem aos produtores identificar as condições climáticas que fragilizam o sistema produtivo. Assim, é uma ferramenta fundamental para o sucesso de um empreendimento agrícola, possibilitando a tomada de decisão no tocante à prática de irrigação para suprir a deficiência hídrica no solo.

De acordo com Matos et al. (2014) é de crucial importância a elaboração do BHC para uma região, já que considera os aspectos relacionados ao solo, a profundidade efetiva do sistema radicular das plantas e a dinâmica de água no solo durante o período avaliado.

Através da estimativa do BHC é possível determinar o período de ocorrência de deficiência e excedente hídrico, retirada e reposição de água do solo e da quantidade de água armazenada no mesmo, por meio dos elementos climáticos mensais, tidos como entrada do modelo, temperatura do ar e precipitação pluvial (CARVALHO et al., 2011).

O sistema de classificação climática de Thornthwaite (1948) permite caracterizar, de forma eficaz, o clima de uma região, devido à metodologia ser sensível aos totais de chuva, e temperatura, somada a informações geradas do BHC (ROLIM, 2007). A delimitação do clima permite estabelecer os indicadores do potencial do meio físico para a região em estudo e determinar áreas homogêneas sob a perspectiva socioeconômica (MEDEIROS et al., 2015a).

A metodologia de cálculo do BHC de Thornthwaite e Mather (1955) e classificação climática de Thornthwaite (1948) são bastante difundidas. Souza et al. (2013) estimaram o BHC e efetuaram a classificação climática para doze municípios do Mato Grosso. Medeiros et al. (2013) calcularam o BHC, realizaram a classificação climática e efetuaram o zoneamento agroclimático para as culturas exploradas no município de Amarante-PI. Medeiros et al. (2015b) realizaram a caracterização agroclimática e a aptidão de culturas para diversos municípios da Paraíba.

Objetivou-se com este estudo desenvolver o balanço hídrico climatológico proposto por Thornthwaite e Mather (1955), para o município de Balsas-MA, para uma capacidade de água disponível no solo (CAD) de $100 \mathrm{~mm}$ e a classificação climática segundo Thornthwaite (1948).

\section{MATERIAL E MÉTODOS}

O balanço hídrico climatológico (BHC) e a caracterização do clima foram realizados para a cidade Balsas-MA (Figura 1). As coordenadas geográficas do local são $7^{\circ} 31^{\prime}$ de latitude sul, $46^{\circ} 2^{\prime}$ de longitude oeste e $243 \mathrm{~m}$ de altitude média. $\mathrm{O}$ município de Balsas localiza-se na parte sul do Maranhão, integrando a microrregião dos Gerais de Balsas, sendo Balsas a cidade principal. Limita-se com os municípios Riachão, São Raimundo das Mangabeiras, Fortaleza dos Nogueiras, Tasso Fragoso, Alto Parnaíba, Nova Colinas, Sambaíba e RecursolândiaTO. Possui uma área de 13.141,733 km², uma população estimada de aproximadamente 93,511 habitantes e uma densidade demográfica de 6,36 habitantes $\mathrm{km}^{-2}$ segundo dados do IBGE (2016). 
REVISTA SCIENTIA AGRARIA

Versão On-line ISSN 1983-2443

Versão Impressa ISSN 1519-1125

SA vol. $18 \mathrm{n}^{\circ} .1$ Curitiba Jan/Mar. 2017 p. 83-89

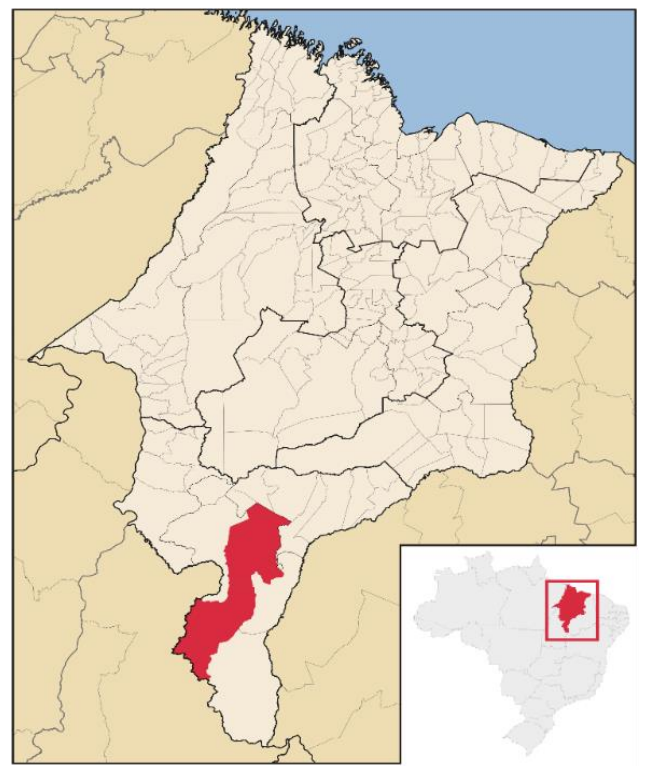

Figura 1: Mapa de localização do município de Balsas-MA.

O clima, segundo a classificação de Köppen, é do tipo tropical quente e úmido (Aw), com estação seca (MARANHÃO, 2002).

O cálculo do BHC foi efetuado adotando-se o valor de $100 \mathrm{~mm}$ para a capacidade de água disponível no solo (CAD); os valores médios mensais de temperatura e precipitação pluvial foram calculados no período de 1976 a 2015. Os dados foram obtidos a partir da estação meteorológica convencional do Instituto Nacional de Meteorologia (INMET), cujo código da Organização Mundial de Meteorologia (OMM) é 82768. A estação localiza-se nas coordenadas $7^{\circ} 31^{\prime}$ de latitude sul, $46^{\circ} 1^{\prime}$ ' de longitude oeste e altitude de $259,38 \mathrm{~m}$. A evapotranspiração potencial (ETP) foi estimada pelo método de Thornthwaite (1948).

Utilizando-se os valores de precipitação pluvial e ETP foi possível estimar a evapotranspiração real (ETR), o armazenamento de água do solo (ARM), a deficiência hídrica (DEF) e o excedente hídrico (EXC).

A classificação climática foi realizada por meio dos valores dos índices: hídrico (Ih), de aridez (Ia) e de umidade (Iu), obtidos conforme equações 1, 2 e 3, respectivamente.

$$
\begin{gathered}
I b=\frac{E X C}{E T P} 100 \\
I a=\frac{D E F}{E T P} 100 \\
I u=I b-I a
\end{gathered}
$$

em que,

EXC - excedente hídrico, em mm;

ETP - evapotranspiração potencial anual, em mm;

DEF - deficiência hídrica, em mm;

Ih- índice hídrico;

Ia - índice de aridez;

Iu - índice de umidade.

\section{RESULTADOS E DISCUSSÃO}

Os resultados obtidos no balanço hídrico climatológico (BHC) são exibidos na Tabela 1, onde verifica-se a variabilidade anual dos elementos climatológicos médios mensais de entrada, temperatura $\left(\mathrm{T} ;{ }^{\circ} \mathrm{C}\right)$ e precipitação pluvial $(\mathrm{P} ; \mathrm{mm})$ e os demais componentes do BHC, evapotranspiração potencial (ETP; mm), armazenamento de água no solo (ARM; $\mathrm{mm}$ ), evapotranspiração real (ETR; $\mathrm{mm}$ ), excedente hídrico (EXC; mm) e deficiência hídrica (DEF; mm). 
REVISTA SCIENTIA AGRARIA

Versão On-line ISSN 1983-2443

Versão Impressa ISSN 1519-1125

SA vol. $18 \mathrm{n}^{\circ} .1$ Curitiba Jan/Mar. 2017 p. 83-89

Tabela 1. Balanço hídrico climatológico pelo método de Thornthwaite e Mather (1955) no período de 1976 a 2015, Balsas-MA.

\begin{tabular}{|c|c|c|c|c|c|c|c|c|c|c|}
\hline Mês & $\begin{array}{c}\mathrm{T} \\
\left({ }^{\circ} \mathrm{C}\right)\end{array}$ & $\begin{array}{c}\mathbf{P} \\
(\mathrm{mm})\end{array}$ & $\begin{array}{l}\text { ETP } \\
(\mathrm{mm})\end{array}$ & $\begin{array}{c}\text { P-ETP } \\
(\mathrm{mm})\end{array}$ & $\begin{array}{c}\text { NEG.AC } \\
(\mathrm{mm})\end{array}$ & $\begin{array}{l}\text { ARM } \\
(\mathrm{mm})\end{array}$ & $\begin{array}{l}\text { ALT } \\
(\mathrm{mm})\end{array}$ & $\begin{array}{c}\text { ETR } \\
(\mathrm{mm})\end{array}$ & $\begin{array}{l}\text { DEF } \\
(\mathrm{mm})\end{array}$ & $\begin{array}{l}\text { EXC } \\
(\mathrm{mm})\end{array}$ \\
\hline Jan. & 26,4 & 199 & 140 & 59 & -16 & 85 & 58 & 140 & 0 & 0 \\
\hline Fev. & 26,6 & 174 & 131 & 43 & 0 & 100 & 15 & 131 & 0 & 28 \\
\hline Mar. & 26,8 & 192 & 145 & 47 & 0 & 100 & 0 & 145 & 0 & 47 \\
\hline Abr. & 27,1 & 124 & 141 & -17 & -17 & 84 & -16 & 140 & 1 & 0 \\
\hline Maio & 27,2 & 45 & 143 & -98 & -115 & 32 & -52 & 97 & 46 & 0 \\
\hline Jun. & 26,7 & 5 & 133 & -128 & -243 & 9 & -23 & 28 & 105 & 0 \\
\hline Jul. & 26,5 & 2 & 133 & -131 & -374 & 2 & -7 & 9 & 124 & 0 \\
\hline Ago. & 27,6 & 4 & 148 & -144 & -518 & 1 & -1 & 5 & 143 & 0 \\
\hline Set. & 28,8 & 27 & 156 & -129 & -647 & 0 & -1 & 28 & 128 & 0 \\
\hline Out. & 28,4 & 90 & 161 & -71 & -718 & 0 & 0 & 90 & 71 & 0 \\
\hline Nov. & 26,7 & 140 & 143 & -3 & -721 & 0 & 0 & 140 & 3 & 0 \\
\hline Dez. & 26,7 & 175 & 148 & 27 & -132 & 27 & 27 & 148 & 0 & 0 \\
\hline Ano & 27,1 & 1175 & 1720 & -545 & - & 440 & 0 & 1099 & 621 & 75 \\
\hline
\end{tabular}

T - Temperatura do ar; P - Precipitação; ETP - Evapotranspiração Potencial; P-ETP - Quantidade de água que permanece no solo; NEG.AC - Negativo Acumulado; ARM - Armazenamento de água no solo; ALT - ARMatual - ARManterior; ETR - Evapotranspiração real; DEF - Deficiência Hídrica e EXC - Excedente Hídrico.

Os meses com a temperatura média mensal mais elevada são setembro e outubro, com 28,8 e $28,4{ }^{\circ} \mathrm{C}$, respectivamente, e o mês mais frio, janeiro com média térmica de $26,4^{\circ} \mathrm{C}$. A temperatura média anual estimada para o município é $27,1{ }^{\circ} \mathrm{C}$, evidenciando pequena variabilidade. Passos et al. (2016) realizaram o cálculo do BHC e classificação climática para uma determinada região de Chapadinha-MA e verificaram que nesse município as temperaturas médias mensais mais elevadas encontram-se nos meses de outubro e novembro, ambos com $29,3^{\circ} \mathrm{C}$ e, mais frio, o mês de junho, com média térmica de $26,9^{\circ} \mathrm{C}$ e a média anual de $27,9^{\circ} \mathrm{C}$.

Constata-se que o município atinge taxas evapotranspirativas mensais bem expressivas. A taxa anual média de ETP total foi de 1720 mm, com variações de 131 $\mathrm{mm}$ no mês de fevereiro a $161 \mathrm{~mm}$ no mês de outubro. Assim, verifica-se o potencial de evapotranspiração maior do que as precipitações. De acordo com Araújo (2011), é por esse motivo que geralmente o balanço hídrico das cidades de climas mais secos da região Nordeste apresentam deficiência hídrica.

Medeiros et al. (2013b) efetuaram classificação climática e zoneamento agroclimático para o município de Amarante-PI. Os autores também observaram altas taxas evapotranspirativas sendo que a ETP anual média do município foi de 1845,4 mm, com variações de $114,5 \mathrm{~mm}$ no mês de fevereiro a 205,5 $\mathrm{mm}$ no mês de outubro.

A precipitação pluvial varia de forma característica durante os meses, atingindo valores médios anuais de 1175 mm (Figura 2). De acordo com a série avaliada, observouse que 1985 foi o ano mais chuvoso, com 1999 mm, e 1982 o ano menos chuvoso, com 566,5 mm. Araújo et al. (2001) observou, para Boa Vista, RR, uma precipitação anual média de $1688,4 \mathrm{~mm}$, sendo 1945 o ano mais chuvoso, com 2554,6 mm, e 1983 o ano menos chuvoso, com 994,7 mm. Verificou-se que em apenas 5 anos a precipitação ultrapassou $1500 \mathrm{~mm}$ e em 18 anos observou-se intensidade acima da média calculada para o período.

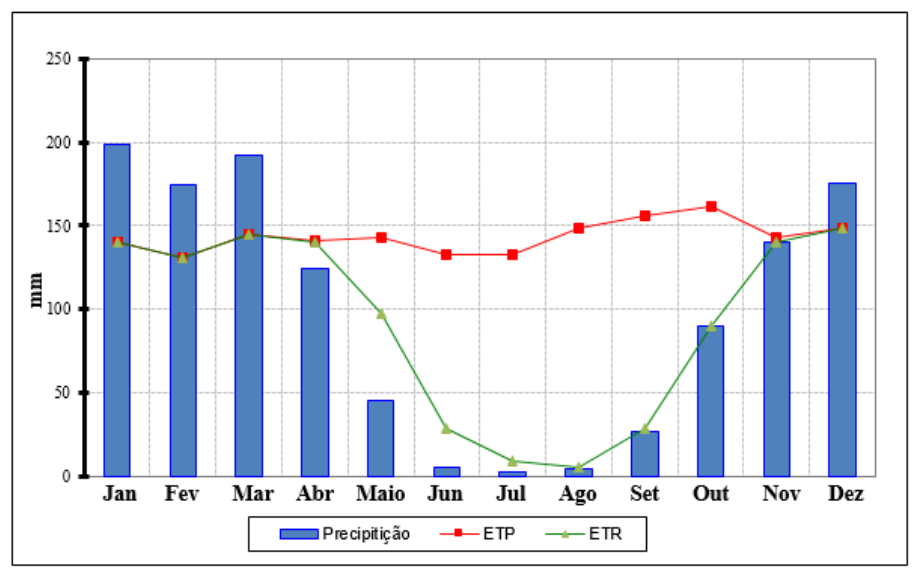

Figura 2: Gráfico do balanço hídrico de Balsas-MA, pelo método de Thornthwaite e Mather (1955), período de 1976 a 2015. 
Verifica-se ainda que a lâmina precipitada é mais elevada ao longo dos meses de novembro a abril, concentrando cerca de $85 \%$ do total acumulado. Já entre os meses de maio e outubro concentra-se aproximadamente $15 \%$ da precipitação pluvial anual. Foi obtido para o mês de janeiro o índice médio mais elevado (199 mm) e o mês de julho o menor ( $2 \mathrm{~mm})$. Menezes et al. (2010) afirmam que a grande variabilidade anual e interanual da precipitação pluvial são características marcantes na região Nordeste do Brasil, o que acarreta prejuízos econômicos e sociais aos estados.

Para o município de Barbalha-CE, Medeiros et al. (2013a) observaram uma média anual da precipitação de 1075,8 mm e que o município apresenta duas estações, uma chuvosa, que responde por $89,46 \%$ do total acumulado e outra seca com apenas 10,53\% do total. Os mesmos autores ainda observaram o mês de março como o mais chuvoso para o período estudado com $227,1 \mathrm{~mm}$ e o mês de agosto o menos chuvoso com apenas 5,8 mm. Medeiros et al. (2013b) averiguaram que para o município de Amarante-PI, o mês de março também possui o índice pluvial médio mensal mais elevado com $281,4 \mathrm{~mm}$ e o mês de agosto o menor índice, 3,6 mm.
Em relação ao armazenamento de água no solo, observou-se que nos meses de janeiro a maio têm-se os maiores índices de armazenamento, variando entre 32 a 100 $\mathrm{mm}$. Nos meses de junho a dezembro a taxa de armazenamento flutua entre 0 a $27 \mathrm{~mm}$, não sendo suficiente à contribuição agrícola.

Em abril, inicia-se o processo de retirada de água do solo que, por conseguinte, resulta na ocorrência de deficiência hídrica na região, compreendida entre os meses de abril e novembro (Figura 3). A necessidade do uso de irrigação suplementar na cidade de Balsas é evidente, pois a região passa por oito meses com deficiência hídrica. Medeiros et al. (2013a) observou que a retirada de água no solo ocorre entre os meses de maio até os primeiros dias do mês de setembro, no município de Barbalha-CE.

Nos meses de fevereiro e março, em virtude das precipitações elevadas, observa-se a ocorrência de excedente hídrico, com 28 e $47 \mathrm{~mm}$, respectivamente, totalizando $75 \mathrm{~mm}$. Nesse período, o solo teoricamente tem umidade apropriada ao crescimento e ao desenvolvimento das culturas agrícolas.

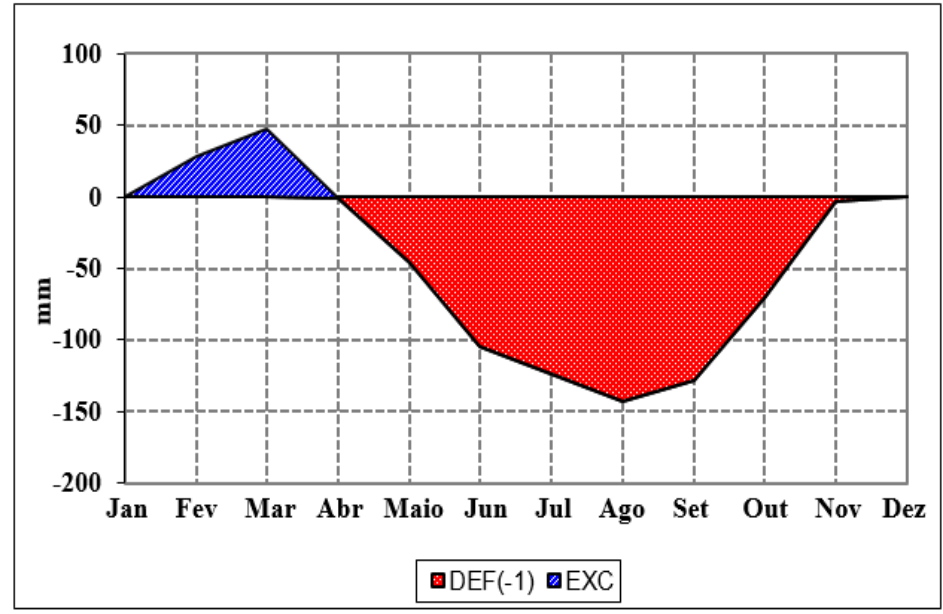

Figura 3: Deficiência hídrica e excedente hídrico de 1976 a 2015.

No início do período úmido, após as chuvas superarem a demanda de evapotranspiração, ou seja, a ETP, tornando essa, igual à evapotranspiração real, os saldos são empregados na reposição da água ao solo (Figura 4) até que esse atinja sua capacidade máxima de armazenamento de água, quando então aparece excedente hídrico. A época de recarga do solo foi observada nos meses de dezembro, janeiro e fevereiro. Para o município de Chapadinha-MA, Passos et al. (2016) constataram que o período de reposição da água do solo ocorre nos meses de janeiro e fevereiro. 


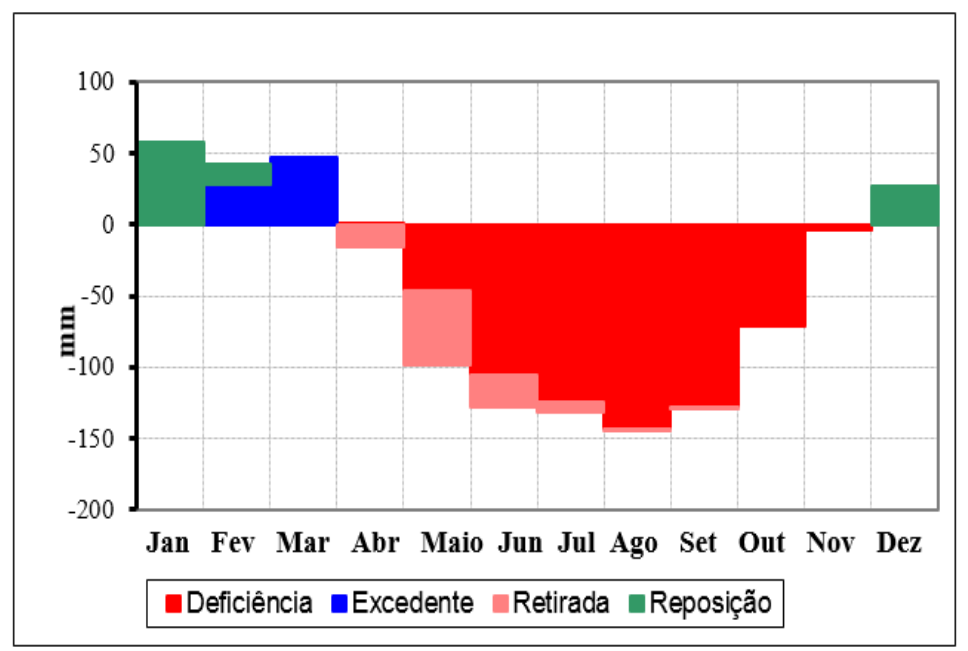

Figura 4: Extrato do balanço hídrico normal mensal, para o período de 1976 a 2015.

A primeira chave da classificação climática foi obtida com base no índice de umidade (Iu), para qual foi encontrado a tipologia $C_{1}$ indicando clima subúmido seco. Com base nos índices de aridez (Ia) e hídrico (Ih), determinou-se o subtipo d, que indica excedente hídrico pequeno ou nulo.

Quanto ao fator térmico, determinado por meio da evapotranspiração potencial anual (ETP), verificou-se que a região estudada, é do tipo A', ou seja, Megatérmico. A partir da relação entre a evapotranspiração potencial de verão e a evapotranspiração potencial anual, obteve-se o subtipo a'.

Assim, a fórmula climática completa obtida foi $\mathbf{C}_{1} \mathbf{d} \mathbf{A} \mathbf{\prime} \mathbf{a}^{\prime}$, ou seja, clima subsumido seco, Megatérmico, com excedente hídrico pequeno ou nulo e com $27 \%$ da evapotranspiração anual concentrada no trimestre mais quente do ano.

\section{CONCLUSÃO}

Para a estação avaliada verificou-se que as precipitações totais anuais atingem valores médios de 1175 $\mathrm{mm}$. De acordo com as informações do BHC, obteve-se a classificação climática para o município de Balsas-MA, como sendo clima subúmido seco, Megatérmico, com excedente hídrico pequeno ou nulo e com $27 \%$ da evapotranspiração anual concentrada no trimestre mais quente do ano, $\mathrm{C}_{1} \mathrm{dA}$ 'a'. A época de excedente hídrico está totalmente compreendida nos meses de fevereiro e março. Já no período de abril a novembro ocorre deficiência hídrica.

\section{REFERÊNCIAS}

ARAÚJO, S. M. S. A Região semiárida do nordeste do Brasil: Questões Ambientais e Possibilidades de uso Sustentável dos Recursos. Rios Eletrônica, v.5, n.5, p.89-98, 2011.

ARAÚJO, W.F.; ADRADE-JUNIOR, A.S.; MEDEIROS, R.S.; SAMPAIO, R.A. Precipitação pluviométrica mensal provável em Boa Vista, Estado de Roraima, Brasil. Revista Brasileira de Engenharia Agrícola e Ambiental, v.5, n.3, p.563-567, 2001.

BLAIN, G.C. Consideraçoes estatísticas relativas à oito séries de precipitação pluvial da secretaria de agricultura e abastecimento do Estado de São Paulo. Revista Brasileira de Meteorologia, v.24, p.12-23, 2009.

CARVALHO, H. P.; DOURADO NETO, D.; TEODORO, R. E. F. MELO, B. Balanço hídrico climatológico, armazenamento efetivo da água no solo e transpiração na cultura de café. Biociência Jornal, v.27, n.2, p.221-229, 2011.

CASTRO, C. N. A agricultura no Nordeste brasileiro: oportunidades e limitações ao desenvolvimento. BRASÍLIA: IPEA, 2012 (Texto para Discussão do IPEA n. 1786).

IBGE. Instituto Brasileiro de Geografia e Estatística. Disponível em:<http://cidades.ibge.gov.br/xtras/perfil.php?codmun $=210140>$. Acesso em: 17/09/2016.

MARANHÃO - GOVERNO DO ESTADO DO MARANHÃO. Gerência de Planejamento e Desenvolvimento Econômico - GEPLAN. Atlas do Maranhão. São Luís: Universidade Estadual do Maranhão, 39 p. 2002.

MATOS, R. M.; SILVA, J. A. S.; MEDEIROS, R. M. Aptidão climática para a cultura do feijão caupi do município de Barbalha - CE. Revista Brasileira de Agricultura Irrigada, v.8, n.6, p.422-431, 2014. 
REVISTA SCIENTIA AGRARIA

Versão On-line ISSN 1983-2443

Versão Impressa ISSN 1519-1125

SA vol. $18 \mathrm{n}^{\circ} .1$ Curitiba Jan/Mar. 2017 p. 83-89

MEDEIROS, R. M.; MATOS, R. M.; SIVA, P. F.; SABOYA, L. M. F.; FRANCISCO, P.R. M. CLASSIFICACÃO CLIMÁTICA E ZONEAMENTO AGROCLIMÁTICO DE CULTURAS PARA SÃO JO ÃO DO CARIRI - PB. Enciclopédia Biosfera, v.11, n.21, p.2984-2996, 2015a.

MEDEIROS, R.M.; SILVA, J.A.S.; SILVA, A. O.; MATOS, R.M.; BALBINO, D.P. BALANCO HÍDRICO CLIMATOLÓGICO E CLASSIFICACÃO CLIMÁTICA PARA A ÁREA PRODUTORA DA BANANA DO MUNICÍPIO DE BARBALHA, CE. Revista Brasileira de Agricultura Irrigada, v.7, n.4, p.258-268, 2013a.

MEDEIROS, R. M.; AZEVEDO, P. V.; SABOYA, L. M. F.; FRANCISCO, P. R. M. CLASSIFICACÃO CLIMÁTICA E ZONEAMENTO AGROCLIMÁTICO PARA O MUNICÍPIO DE AMARANTE - PI. Revista Brasileira de Agricultura Irrigada, v.7, n.2, p.170-180, 2013b.

MEDEIROS, R.M.; FRANCISCO, P. R. M.; MATOS, R. M.; SANTOS, D.; SOUSA, T.P. Caracterização agroclimática e aptidão de culturas para diferentes municípios e regiões da Paraíba. Agropecuária Científica no Semi-Árido, v.11, n.2, p.99-110, 2015b.

MENEZES, H. E. A.; DE BRITO, J. I. B.; LIMA, R. A. F. A. Veranico e a produção agrícola no Estado da Paraíba, Brasil. Revista Brasileira de Engenharia Agrícola e Ambiental, v.14, n.2, p.181-186, 2010.

PASSOS, M.L.V.; ZAMBRZYCKI, G.C.; PEREIRA, R.S. BALANCO HÍDRICO E CLASSIFICACÃO CLIMÁTICA PARA UMA DETERMINADA REGLÃO DE CHAPADINHA-MA. Revista Brasileira de Agricultura Irrigada, v.10, n.4, p.758-766, 2016.

ROLIM, G. S. Classificação climática de Köppen e de Thornthwaite e sua aplicabilidade na determinação de zonas agroclimáticas para o estado de São Paulo. Revista Bragantia, v.66, n.4, p.711-720, 2007.

SANTOS, G. O.; HERNANDEZ, F. B. T.; ROSSETTTI, J. C. BALANCO HÍDRICO COMO FERRAMENTA AO PLANEJAMENTO AGROPECUÁRIO PARA A REGIÄO DE MARINÓPOLIS, NOROESTE DO ESTADO DE SÃO PAULO. Revista Brasileira de Agricultura Irrigada, v.4, n.3, p.142-149, 2010.

SOUZA, A.P.; MOTA, L.L.; ZAMADEI, T.; MARTIM, C.C.; ALMEIDA, F.T.; PAULINO, J. CLASSIFICAC $\tilde{A O}$ CLIMÁTICA E BALANCO HÍDRICO CLIMATOLÓGICO NO ESTADO DE MATO GROSSO. Nativa, Sinop, v.1, n.1, p.34-43, 2013.

THORTHWAITE, C.W.; MATHER, J.R. The water balance. Publications in Climatology. New Jersey: Drexel Institute of Technology; 1955, 104p.
THORTHWAITE, C. W. An approach towards a rational classification of climate. Geographical Review, London, v.38, p.55-94, 1948. 